\title{
The comparison of chest CT and RT-PCR during the diagnosis of COVID-19
}

\author{
Avni Uygar Seyhan ${ }^{1}$, Fatih Doğanay ${ }^{2}$, Erdal Yılmaz', Ömer Aydıner3, Rohat Ak ${ }^{1}$, Serap Demir Tekol ${ }^{4}$ \\ ${ }^{1}$ Department of Emergency Medicine, Kartal Dr. Lütfi Kırdar City Hospital, Istanbul, Turkey \\ ${ }^{2}$ Department of Emergency Medicine, Edremit Public Hospital, Ballkesir, Turkey \\ ${ }^{3}$ Department of Radiology, Kartal Dr. Lütfi Kirdar City Hospital, Istanbul, Turkey \\ ${ }^{4}$ Department of Microbiology, Kartal Dr. Lütfi Kırdar City Hospital, Istanbul, Turkey
}

Received: 2020-10-14.

Accepted: $2020-11-28$

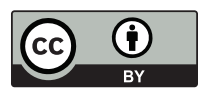

This work is licensed under a Creative Commons Attribution 4.0 International License

\section{J Clin Med Kaz 2021; 18(1):53-56}

\section{Corresponding author:}

Rohat Ak.

E-mail: rohatakmd@gmail.com;

ORCID: 0000-0002-8324-3264

\section{Abstract}

Objective: Our aim in this study is to compare the diagnostic accuracy of chest computed tomography and Real-time Reverse Transcriptase Polymerase Chain Reaction in a population with suspected Coronavirus Disease 2019 (COVID-19) presenting at the emergency department.

Material and methods: The study included 257 patients presented to the emergency department with suspected COVID-19. We included all symptomatic patients who received a chest computed tomography and at least one Polymerase Chain Reaction test for detection of COVID-19. The detection rate of COVID-19 infection based on the initial chest computed tomography and Real-time Reverse Transcriptase Polymerase Chain Reaction was compared.

Results: A total of 257 patients, 130 women and 127 men, were included in the study. The average age of all patients was 41,12 $\pm 15,77$. Sensitivity of Real-time Reverse Transcriptase Polymerase Chain Reaction test was $71 \%$ while that of computed tomography was found to be $49 \%$. The ground glass appearance and/or peripheral distribution findings in 126 patients can be said to be the representative of computed tomography findings for COVID-19.

Conclusion: Chest computed tomography should not be used routinely as a screening or diagnostic tool for COVID-19, and additional risks that may arise due to computed tomography imaging should be considered.

Key words: coronavirus, reverse transcription polymerase chain reaction, chest $\mathrm{CT}$ imaging

\section{Introduction}

COVID-19 (coronavirus disease 2019) is an infectious disease that is caused by the severe Acute Respiratory Syndrome Coronavirus 2 (SARS-CoV-2), a coronavirus strain. Before spreading globally, the first cases were detected in Wuhan, China, in December 2019, and now more than 25 million cases have been confirmed. The current outbreak was officially recognized as an epidemic by the World Health Organization (WHO) on March 11, 2020 [1-3].

Real-time Reverse Transcriptase Polymerase Chain Reaction (RT-PCR) demonstrating SARS-CoV-2 RNA in respiratory system samples is the gold standard method for confirming the diagnosis of infection. Although the test is said to be highly specific, its sensitivity varies between $60-89 \%$ [4].

In the early stages of the Covid-19 outbreak, many studies have been reported that chest Computed Tomography (CT) is more successful than RT-PCR in the diagnosis of the disease [5,6]. However, recently, radiological communities have stated that $\mathrm{CT}$ should not be relied upon as a diagnostic or screening tool for COVID-19 [7-9].

In this study, we investigated the diagnostic accuracy of Chest CT scanning in detecting COVID-19 in a population with suspected COVID-19 presenting at the emergency department (ED) using (repeated) RT-PCR testing as reference standard.

\section{Material and methods}

Study design and selection of patients

This retrospective observational study was carried out in the ED of a tertiary care teaching hospital between April 1, 2020 and May 31, 2020. The institutional review 
board approved the analysis and issued a waiver of consent (Ethics Committee Ruling number: 2020/514/178/6). All adult (18 years or older) patients who visited the ED between March 1 and May 31, 2020 with fever and respiratory symptoms including dyspnea, coughing, sore throat were scanned in a chest CT scan unit. Of these patients, a nasopharyngeal and/or oropharyngeal swab was taken and tested for presence of SARS-CoV-2. If the first PCR was negative, a second, third, fourth and fifth PCR was performed within 48 hours after the first test in patients who were still admitted to the hospital, if deemed indicated by the clinicians. We included all symptomatic patients who received a chest CT and at least one PCR test for detection of COVID-19.

\section{Chest CT protocols and Image analysis}

All CT examinations for the screening of SARS-CoV-2 pneumonia were performed with three scanners (128 section Philips ingenuity and 16-section Toshiba Alexion without the use of contrast material. The main scanning protocol was as follows: tube voltage, $120 \mathrm{kVp}$; tube current modulation, $120 \mathrm{~mA}-380$ $\mathrm{mA}$; detector configuration, $64 \times 0.625 \mathrm{~mm}$ or $16 \times 0.625 \mathrm{~mm}$; rotation time, $0.5-0.7 \mathrm{~s}$; slice thickness, $5 \mathrm{~mm}$; and pitch, 0.984 . Reconstruction kernel was lung with a thickness and an interval of $0.625 \mathrm{~mm}$. All images we reviewed in both lung (width, 1200 HU; level, - $700 \mathrm{HU}$ ) and mediastinal (width, $350 \mathrm{HU}$; level, 40 HU) settings.

The radiologist (Ö.A) with 20 years of experiences who was blinded to the other clinical information reviewed the chest CT scans independently and then reached a decision.

The images were interpreted using the lung window setting. The CT images were assessed, following a standardized protocol, for the presence and distribution of the following abnormalities: (a) ground-glass opacities (GGO) defined as hazy areas of increased attenuation without obscuration of the underlying vascular markings); (b) nodules (centrilobular, perilymphatic, or random in distribution); (c) linear densities (interlobular septal thickening, intralobular septal line, parenchymal bands); (d) crazy paving; (e) consolidations (parenchymal opacities obscuring underlying vessels); (f) architectural distortion, or traction bronchiectasis; (g) pleural effusion; (h) lymphadenopathy (defined as lymph node with a short-axis dimension of $>1.0 \mathrm{~cm}$ ); (i) air bronchogram; (j) treein-bud sign (defined as multiple areas of centrilobular nodules with a linear branching pattern); and (k) white lung (defined as diffuse consolidations in a large area of the lung that appear like the lung is turning white on CT imaging). Typical and atypical chest CT findings were recorded according to CT features previously described for COVID-19 [10]. If there is at least one of these findings, the case was accepted as "CT positive for COVID-19". The overall anatomic distribution (subsegmental, segmental, lobar), zonal predominance (upper, middle, lower lung; central, middle, or peripheral location), and extent (focal, multifocal, and diffuse) of the lesions were also recorded.

\section{RT-PCR}

Nasopharyngeal/oropharyngeal specimens were collected with sterile synthetic fiber swabs with plastic shafts and were placed in Viral Transport Medium. Collected specimens were transported to the Molecular Microbiology Diagnosis Laboratory within $12 \mathrm{~h}$ and tested within $9 \mathrm{~h}$ after laboratory acceptance. Detection of SARS-CoV-2 in specimens was performed by Real Time Reverse Transcriptase Polymerase Chain Reaction (RTPCR) test by using The Direct Detect SARS-CoV-2 Detection Kit (Coyote Bioscience Co., Ltd). This kit targets the ORF

$1 \mathrm{ab}$ and $\mathrm{N}$ gene of SARS-CoV-2, covered by a pair of specific primers and a fluorescence-labeled probe. Swab specimens in VTM were vortexed for $15 \mathrm{~s}$ and then $15 \mathrm{uL}$ of the sample was transferred to a sterile microcentrifuge tube. A 15 uL Respiratory Sample Buffer (Base Lye solution) added into the tube and lysis step was performed according to the manufacturer's instructions. $15 \mathrm{uL}$ sample-buffer mixture pipetted in to amplification Reagent. Amplification Reagent was prepared by following manufacturer's instructions and consists of ORF $1 \mathrm{ab}, \mathrm{N}$ gene of SARS-CoV-2, RNase P gene of human detection, reverse transcriptase, Taq DNA Polymerase deoxyribo triphosphates (dNTP), $\mathrm{MgCl} 2$ and other buffers. A negative (Hank's sample storage solution and RNP Pseudo virus) control, a non-template (water) control and a positive control was included in every RT-PCR run. PCR amplification was performed on Bio-Rad CFX96 Deep Well Real-Time PCR Detection System (Bio-Rad Laboratories). Cycle threshold (Ct) values of less than 29 were defined as positive. Limit of detection of the kit was decelerated as 500 copies $/ \mathrm{mL}$ for both the ORF 1 ab gene and $\mathrm{N}$ gene by manufacturer. The detection rate of COVID-19 infection based on the initial chest CT and RT-PCR was compared.

\section{Statistical Analysis}

IBM SPSS Statistics 26.0 software (Chicago, IL) was used for statistical analysis. Quantitative data are expressed as Mean \pm Standard Deviation (SD). Categorical data were compared using the Chi-Square test so expressed as frequency and percentage. As a result of the analysis, when the smallest theoretical frequency value is less than 5, Fisher's Exact test result was reported. A p value less than 0.05 was considered statistically significant.

\section{Results}

After the inclusion and exclusion criteria of the study were applied to the patients, the study was completed with 257 patients. Of the patients included in the study, 130 (50.6\%) were female and $127(49.4 \%)$ were male. The age range of the patients was 18 to 91 . The average age of all patients was $41,12 \pm 15,77$. In the RT-PCR tests performed on 257 patients included in the study, $184(71,6 \%)$ patients had positive results while 73 $(28,4 \%)$ patients were negative. As a result of the second RTPCR of 73 patients with negative results in the first RT-PCR test, $56(76.7 \%)$ patients had positive test results while 17 (23.3) patients had negative results. Repeated RT-PCR test results of patients with negative RT-PCR results and finally diagnosed with Covid-19 are given in Table 1.

Table 1

\begin{tabular}{|l|l|l|l|}
\hline & Total Case & Positive Case & Negative Case \\
\hline Test 1 & 257 & $184(71,6 \%)$ & $73(28,4 \%)$ \\
\hline Test 2 & 73 & $56(76,7 \%)$ & $17(23,3 \%)$ \\
\hline Test 3 & 17 & $12(70,6 \%)$ & $5(29,4 \%)$ \\
\hline Test 4 & 5 & $4(80 \%)$ & $1(20 \%)$ \\
\hline Test 5 & 1 & $1(100 \%)$ & 0 \\
\hline
\end{tabular}

CT imaging findings of 257 patients diagnosed with COVID-19 by RT-PCR are shown in Table 2. Considered CT findings of Covid-19 pneumonia; the following findings were detected : ground glass opacity in 122 patients, consolidation in 47 patients, multiple lesions in 86 patients, bilateral lung involvement in 92 patients, posterior and/or inferior lobe involvement in 83 patients, peripheral involvement in 105 


\begin{tabular}{|c|c|c|c|c|c|}
\hline & \multicolumn{2}{|l|}{$\begin{array}{l}\text { RT-PCR negative } \\
(\mathrm{n}=73)\end{array}$} & \multicolumn{2}{|l|}{$\begin{array}{l}\text { RT-PCR positive } \\
(\mathrm{n}=184)\end{array}$} & \multirow[b]{2}{*}{$\mathrm{p}$ value } \\
\hline & CT Finding NO & CT Finding YES & CT Finding NO & CT Finding YES & \\
\hline Ground glass opacities & $46(63 \%)$ & $27(37 \%)$ & $89(48,4 \%)$ & $95(51,6 \%)$ & 0.04 \\
\hline Consolidation & $58(79,5 \%)$ & $15(20,5 \%)$ & $152(82,6 \%)$ & $32(17,4 \%)$ & 0.68 \\
\hline $\begin{array}{l}\text { Multilobe and Multiple lesions } \\
\text { (involving } 4 \text { or } 5 \text { lobes) }\end{array}$ & $55(75,3 \%)$ & $18(24,7 \%)$ & $116(63 \%)$ & $68(37 \%)$ & 0,08 \\
\hline Bilateral involvement & $52(71,2 \%)$ & $21(28,2 \%)$ & $113(61,4 \%)$ & $71(38,6 \%)$ & 0,18 \\
\hline Posterior / lower lobe involvement & $51(69,9 \%)$ & $22(30,1 \%)$ & $123(66,8 \%)$ & $61(33,2 \%)$ & 0,75 \\
\hline $\begin{array}{l}\text { Peripheral / subpleural distribution } \\
\text { (external 1/3) }\end{array}$ & $47(64,4 \%)$ & $26(35,6 \%)$ & $105(57,1 \%)$ & $79(42,9 \%)$ & 0,34 \\
\hline Crazy Paving & $70(95,9 \%)$ & $3(4,1 \%)$ & $173(94 \%)$ & $11(6 \%)$ & $0,76^{*}$ \\
\hline Air bronchogram & $62(84,9 \%)$ & $11(15,1 \%)$ & $158(85,9 \%)$ & $26(14,1 \%)$ & 1,00 \\
\hline Reverse halo sign in CT & $69(94,5 \%)$ & $4(5,5 \%)$ & $171(92,9 \%)$ & $13(7,1 \%)$ & $0,78^{*}$ \\
\hline Pleural effusion & $73(100 \%)$ & $0(0 \%)$ & $181(98,4 \%)$ & $3(1,6 \%)$ & $0,56^{*}$ \\
\hline Vascular enlargement in the lesion & $65(89 \%)$ & $8(11 \%)$ & $166(90,2 \%)$ & $18(9,8 \%)$ & 0,95 \\
\hline At least $1 \mathrm{CT}$ finding & $44(60,3 \%)$ & $29(39,7 \%)$ & $87(47,3 \%)$ & $97(52,7 \%)$ & 0.08 \\
\hline
\end{tabular}

*p value of Fisher'sExact Test

patients, Crazy paving in 14 patients, bronchogram in 37 patients, reverse halo sign in 17 patients, pleural effusion in 3 patients and vascular enlargement in the lesion in 26 patients (Table 2). The total of 126 patients with ground-glass opacity and/or peripheral (subpleural) distribution features was 126, and it seemed to overlap in terms of CT findings with 126 patients with at least one CT finding. It can be said that these two imaging findings are representative of CT findings for Covid-19.

When the CT finding was compared with the RT-PCR result, it was determined that the only imaging finding that was significantly different was the ground glass image. CT images of 257 patients diagnosed with Covid-19 with repeated RTPCR tests were examined at the time of admission, where the first RT-PCR examination was taken. 126 (49\%) patients had at least one abnormal CT finding at presentation. 73 cases received false negative results with the first RT-PCR test. Of these 73 overlooked cases, 56 cases in second RT-PCR test, 12 cases in the third test, 4 cases in the 4 th test, and the remaining 1 case in the 5th test were detected positive. The sensitivity of CT findings was found to be $49 \%$ at the time of presentation, and the sensitivity of the first RT-PCR result performed on the same day with CT was determined as $71.6 \%$. Because of its high clinical suspicion, 29 (40\%) of 73 patients who underwent a second RTPCR test had imaging findings in their CT at the time of first application. When evaluated specifically for the second test, RTPCR sensitivity was $76 \%$, while CT sensitivity was found to be $40 \%$ (Table 3 ).

Table 3

Chi-square test results comparing the CT images with RT-PCR test.

\begin{tabular}{|l|l|l|l|l|l|l|}
\hline \multicolumn{2}{|l|}{} & \multicolumn{2}{l|}{ RT-PCR negative } & \multicolumn{2}{l|}{ RT-PCR positive } & \\
\hline $\begin{array}{l}\text { Test } \\
\text { no }\end{array}$ & $\begin{array}{l}\text { Total } \\
\text { test }\end{array}$ & $\begin{array}{l}\text { CT } \\
\text { Finding } \\
\text { NO }\end{array}$ & $\begin{array}{l}\text { CT } \\
\text { Finding } \\
\text { YES }\end{array}$ & $\begin{array}{l}\text { CT } \\
\text { Finding } \\
\text { NO }\end{array}$ & $\begin{array}{l}\text { CT } \\
\text { Finding } \\
\text { YES }\end{array}$ & P value \\
\hline Test 1 & 257 & $\begin{array}{l}44(60,3 \\
\%)\end{array}$ & $\begin{array}{l}29 \\
(39,7 \%)\end{array}$ & $\begin{array}{l}87 \\
(47,3 \%)\end{array}$ & $\begin{array}{l}97 \\
(52,7 \%)\end{array}$ & 0.082 \\
\hline Test 2 & 73 & $\begin{array}{l}11 \\
(64,7 \%)\end{array}$ & $\begin{array}{l}6 \\
(35,3 \%)\end{array}$ & $\begin{array}{l}33 \\
(58,9 \%)\end{array}$ & $\begin{array}{l}23 \\
(41,1 \%)\end{array}$ & 0.88 \\
\hline Test 3 & 17 & $3(60 \%)$ & $2(40 \%)$ & $8(66,7 \%)$ & $\begin{array}{l}4 \\
(33,3 \%)\end{array}$ & $1,00^{*}$ \\
\hline
\end{tabular}

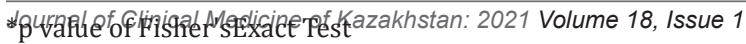

\section{Discussion}

Due to the strong infectivity profile of COVID-19, early diagnosis and treatment are very important in the management of patients, otherwise human-induced disease spread could seriously endanger public health. However, the routine use of $\mathrm{CT}$ as a diagnostic or screening tool carries many risks like the excessive use and consumption of personal protective equipment (PPE), ionizing radiation exposures and increased risk of viral transmission [11].

In our study, the data of 257 patients were analyzed retrospectively and the sensitivity of RT-PCR test was found to be higher than CT (71.6\% vs. 49\%). In addition, ground glass appearance and/or the peripheral distribution findings that were detected in 126 patients can be considered as the two representative CT findings for COVID-19. COVID-19 pneumonia series was first reported in China. Based on a prospective analysis of 1014 patients, it was reported that the sensitivity of CT in detecting COVID-19 was $97 \%$, and the sensitivity of RT-PCR ranged from $60 \%$ to $70 \%$ [5]. In another study conducted in China, it was stated that COVID-19 pneumonia can manifest itself with chest CT imaging abnormalities even in asymptomatic patients, and combining imaging features with clinical and laboratory findings will facilitate the early diagnosis of COVID-19 pneumonia [12].

In a study conducted in Italy, the typical patterns of COVID-19 pneumonia were detected as multilobe and posterior involvement, bilateral distribution, subsegmental vasodilation (>3 mm) and peripheral ground glass opacities, and the sensitivity of chest CT was 97\% [13]. In countries with a low COVID prevalence $(<10 \%)$, the positive predictive value of RT-PCR has been reported to be ten times that of chest CT [4]. Recently, in a meta-analysis covering a wide prevalence range, RT-PCR sensitivity was reported to be $94 \%$ and specificity $37 \%$ [14]. As seen in the literature, for COVID-19, the sensitivity and specificity of RT-PCR and chest CT are controversial.

In our study, the sensitivity of the RT-PCR test was $71 \%$, and that of CT was $49 \%$. We think that the conducted studies which were independent of the patients' clinical presentation and were consisted of only CT and RT-PCR comparisons led to the difference in sensitivity and specificity values. The Fleischner Association has issued a consensus statement that aims to guide 
the use of patient management and imaging methods during the COVID-19 outbreak. According to this; 1) Routine imaging is not required as a screening test for COVID-19 in asymptomatic cases. 2) There is no need for imaging in COVID-19 patients with mild clinical findings unless they are at risk for disease progression. 3) Imaging is required regardless of test results in the presence of moderate or severe clinical findings consistent with COVID-19. 4) Imaging is required whenever respiratory symptoms worsen in COVID-19 cases. 5) In resource constraints where access to CT is limited, if there is worsening of respiratory symptoms the use of CT is not required and imaging with direct graph may be preferred. 6) In cases of COVID-19, if there is functional deterioration and/or hypoxemia after recovery, CT is indicated. 7) COVID-19 test should be performed when findings suggesting COVID-19 are detected incidentally in CT examination [15].

\section{Limitations}

Our study had several limitations. Identifying a small number of patients in a single academic institution, this study potentially limits the generalization of results with other populations. In addition, in our study, we compared RT-PCR with CT without knowing the clinical status of the patients and this might unintentionally caused selection bias.

\section{Conclusion}

Chest CT should be used according to the clinic presentation of the patients and to evaluate the complications in some other patients. Its use for screening or as a diagnostic tool for COVID-19 may cause additional risks.

Disclosures: There is no conflict of interest for all authors.

\section{Acknowledgments: None.}

Funding: The author(s) received no financial support for the research, authorship, and/or publication of this article.

\section{References}

1. Na Zhu, Dingyu Zhang, Wenling Wang, Xinwang Li, Bo Yang, Jingdong Song, Xiang Zhao, Baoying Huang, Weifeng Shi, Roujian Lu, Peihua Niu, Faxian Zhan, Xuejun Ma, Dayan Wang, Wenbo Xu, Guizhen Wu, George F Gao, Wenjie Tan. A Novel Coronavirus from Patients with Pneumonia in China, 2019. New England Journal of Medicine. 2020; doi:10.1056/NEJMoa2001017

2. Another Decade, Another Coronavirus. New England Journal of Medicine. 2020; doi:10.1056/NEJMe2001126

3. "WHO Director-General's opening remarks at the media briefing on COVID-19 - 11 March 2020". Who.int, 2020.

4. Fang Y., Zhang H., Xie J., Lin M., Ying L., Pang P., Ji W. Sensitivity of chest CT for COVID-19: comparison to RT-PCR. Radiology. 2020; 200432. https://doi.org/10.1148/radiol.2020200432

5. Ai T., Yang Z., Hou H., Zhan C., Chen C., Lv W., Xia L. Correlation of chest CT and RT-PCR testing in coronavirus disease 2019 (COVID-19) in China: a report of 1014 cases. Radiology. 2020; 200642. https://doi.org/10.1148/radiol.2020200642

6. Long C., Xu H., Shen Q., Zhang X., Fan B., Wang C., Li H. Diagnosis of the Coronavirus disease (COVID-19): rRT-PCR or CT?. European journal of radiology. 2020; 108961. https://doi.org/10.1016/j.ejrad.2020.108961

7. RCR position on the role of CT in patients suspected with COVID-19 infection | The Royal College of Radiologists". Rcr.ac.uk, 2020.

8. "Canadian Society of Thoracic Radiology and Canadian Association of Radiologists' Statement on COVID -19 - CAR - Canadian Association of Radiologists". CAR - Canadian Association of Radiologists, 2020.

9. Rodrigues J. C. L., Hare S. S., Edey A., Devaraj A., Jacob J., Johnstone A., Robinson G. An update on COVID-19 for the radiologist-A British society of Thoracic Imaging statement. Clinical radiology. 2020; 75(5):323-325. https://doi.org/10.1016/j.crad.2020.03.003

10. Pan F., Ye T., Sun P., Gui S., Liang B., Li L., Zheng C. Time course of lung changes on chest CT during recovery from 2019 novel coronavirus (COVID-19) pneumonia. Radiology. 2020; 200370. https://doi.org/10.1148/radiol.2020200370

11. Constantine A. Raptis, Mark M. Hammer, Ryan G. Short, Amar Shah, Sanjeev Bhalla, Andrew J. Bierhals, Peter D. Filev, Michael D. Hope, Jean Jeudy, Seth J. Kligerman, Travis S. Henry. Chest CT and Coronavirus Disease (COVID-19): A Critical Review of the Literature to Date. American Journal of Roentgenology. 2020.

12. Shi H, Han X, Jiang N. Radiological ndings from 81 patients with COVID-19 pneumonia in Wuhan, China: a descriptive study. Lancet Infectious Diseases. 2020; 1-10. doi: 10.1016/ S1473-3099(20)30086-4

13. Caruso D, Zerunian M, Polici M. Chest CT features of COVID-19 in Rome, Italy. Radiology. 2020; 201237. doi: 10.1148/ radiol.2020201237

14. Kim H., Hong H., Yoon S. H. Diagnostic performance of CT and reverse transcriptase-polymerase chain reaction for coronavirus disease 2019: a meta-analysis. Radiology. 2020; 201343. https://doi.org/10.1148/radiol.2020201343

15. Rubin Geoffrey D., Linda B. Haramati and Jeffrey P. Kanne et al. The Role of Chest Imaging in Patient Management during the COVID-19 Pandemic: A Multinational Consensus Statement from the Fleischner Society. Radiology. 2020; 201365. https://doi. org/10.1148/radiol.2020201365 